\title{
Retrosigmoid Approach for Resection of Large Cystic Vestibular Schwannoma
}

\author{
Michael J. Link ${ }^{1,2}$ Colin L. W. Driscoll ${ }^{1,2}$ Yening Feng ${ }^{2}$ Maria Peris-Celda ${ }^{1}$ Christopher S. Graffeo ${ }^{1}$ \\ ${ }^{1}$ Department of Neurologic Surgery, Mayo Clinic, Rochester, \\ Minnesota, United States \\ 2 Department of Otorhinolaryngology, Mayo Clinic, Rochester, \\ Minnesota, United States

\begin{abstract}
Address for correspondence Michael J. Link, MD, Department of Neurosurgery, Department of Otolaryngology-Head and Neck Surgery, Mayo Clinic School of Medicine, Rochester, MN 55905
\end{abstract} \\ (e-mail: link.michael@mayo.edu).
}

J Neurol Surg B 2019;80(suppl S3):S285.

\begin{abstract}
Keywords

- retrosigmoid

- vestibular schwannoma

- acoustic neuroma

- skull base

Objectives This video was aimed to describe the surgical indications, relevant anatomy, and surgical steps of retrosigmoid approach for resection of a large cystic vestibular schwannoma (VS).

Design The operative steps are described in a surgical instructional video.

Setting The surgery took place at a tertiary skull base referral center.

Participant Patient is a 62-year-old man who reported with right sided profound hearing loss with no word recognition, progressive dizziness and tinnitus, excruciating burning pain in the V2 distribution of right trigeminal nerve, wide-based gait, and a right cerebellopontine angle (CPA) cystic VS measuring $3.3 \mathrm{~cm}$.

Main Outcome Measures The large cystic VS was resected through retrosigmoid approach.

Results The surgery resulted in removal of the large cystic VS with initial delayed facial weakness that completely resolved (House Brackmann grade 1) by 3 month follow-up. The patient had no other postoperative complications and is convalescing well from the procedure.

Conclusion Cystic VS presents some unique challenges compared with their solid counterparts. The cystic tumor capsule may be very adherent to the adjacent structures, and distinguishing thin cyst walls from the arachnoid of the CPA, can be quite challenging. The retrosigmoid approach provides adequate surgical exposure for VS tumor resection. The link to the video can be found at: https://youtu.be/sFNvRWG465Q.
\end{abstract}

Conflict of Interest

None.

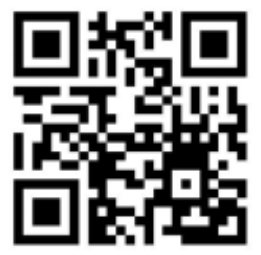

www.thieme.com/skullbasevideos

www.thieme.com/jnlsbvideos

received

May 31, 2018

accepted after revision

November 11, 2018

published online

February 26, 2019
DOI https://doi.org/

10.1055/s-0039-1677850.

ISSN 2193-6331.
๑) 2019 Georg Thieme Verlag KG
Stuttgart · New York

License terms

(c) (1) $\ominus$ (\$) 